\title{
Swarm Decomposition Technique Based Hybrid Model for Very Short-Term Solar PV Power Generation Forecast
}

\author{
Emrah Dokur \\ Department of Electrical Electronics Engineering, Bilecik S. E. University, \\ 11210 Bilecik, Turkey \\ emrah.dokur@bilecik.edu.tr
}

\begin{abstract}
Accurate predictions of solar photovoltaic (PV) power generation at different time horizons are essential for reliable operation of energy management systems. The output power of a PV power plant is dependent on non-linear and intermittent environmental factors, such as solar irradiance, wind speed, relative humidity, etc. Intermittency and randomness of solar PV power effect precision of estimation. To address the challenge, this paper presents a Swarm Decomposition Technique (SWD) based hybrid model as a novel approach for very short-term $(15 \mathrm{~min})$ solar $\mathrm{PV}$ power generation forecast. The original contribution of the study is to investigate use of SWD for solar data forecast. The solar PV power generation data with hourly resolution obtained from the field (grid connected, $857.08 \mathrm{kWp}$ Akgul Solar PV Power Plant in Turkey) are used to develop and validate the forecast model. Specifically, the analysis showed that the hybrid model with SWD technique provides highly accurate predictions in cloudy periods.
\end{abstract}

Index Terms-Energy management; Forecasting; Solar energy; Swarm decomposition.

\section{INTRODUCTION}

Rising energy demand and its related environmental impacts increase the use of renewable energy resources in generation rapidly. Solar photovoltaic PV panels have recently become one of the fastest growing renewable power generation techniques. Renewable energy resources are nonlinear structures and highly dependent on geographical locations and weather conditions. Integration of large-scale photovoltaic power systems into interconnected systems is becoming a major challenge [1].

Since the solar irradiation on the surface shows a highly variable behavior due to cloudiness, it brings difficulties in planning and operation of energy systems with PV power generation [2]. Some significant challenges, such as stability, reliability, supply/demand balance, reactive power compensation and frequency response, in power systems may be faced due to large-scale implementation of grid connected PV solar power plants [3], [4]. Instability in the integration of these systems into the grid and harmonics in power outputs needs to be eliminated. Therefore, the accurate estimation of the energy to be obtained from PV

Manuscript received 25 February, 2020; accepted 18 May, 2020. systems is very important in order to ensure optimum planning and modeling of photovoltaic systems [4]. The generation schedules should be planned for very short-term, short-term and long-term power system operation in order to ensure reliable operation and economic dispatch of modern power systems (Fig. 1).

The researchers have proposed lots of methods with various time horizons for modeling and forecasting of PV power, such as deep learning approaches [5], [6], hybrid models [7], [8], artificial intelligence algorithms [9]-[11], and support vector machines (SVM) [12], [13].

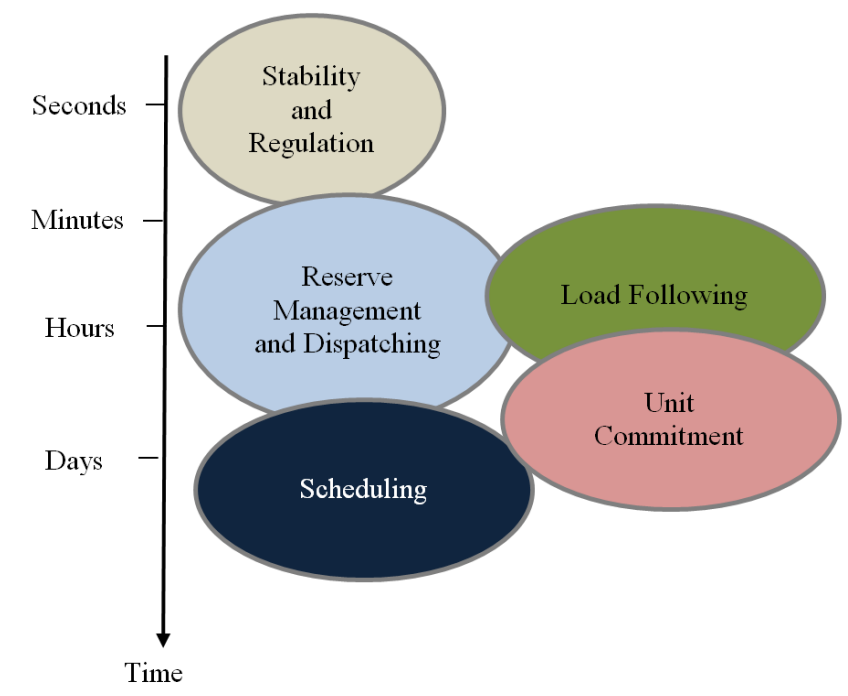

Fig. 1. Forecasting horizon and related applications.

Both weather data (such as wind speed, solar irradiance, humidity, temperature, etc.), and historical power outputs can be used for forecasting models. The use of weather data requires processing of big data, which results in long analysis time in forecasting. Besides, predictive models can operate faster with historical power values. One of the disadvantages of these models is low accuracy during cloudy periods. Different pre-processing techniques have been proposed in the literature to reduce this disadvantage. In [8], a hybrid model based on random vector functional link SARIMA time series analysis and use of wavelet decomposition in pre-processing step for very short-term PV power forecasting was proposed. The data were decomposed 
into several modes in the pre-processing step. In case of having high-frequency components due to cloudy or rainy sky conditions in the solar PV power output, pre-processing step based on decomposition technique made the estimation approach perform better.

In this paper, a novel hybrid approach, swarm decomposition and feed forward neural network (SWDFFNN), has been proposed for very short-term solar power forecasting. The solar power output data obtained from the Akgul- Solar PV Power Plant (SPP) were pre-processed using SWD for detecting cloudy/rainy sky conditions variance. In Section II, SWD technique used in preprocessing is presented. The hybrid approach is explained with a flowchart in Section III. Simulation results are presented in Section IV. Finally, the study is concluded in Section V.

\section{PRE-PROCESSING BASED ON SWARM DECOMPOSITION TECHNIQUE}

Apostolidis and Hadjileontiadis [14] have proposed a novel signal analysis called "swarm decomposition" (SWD). SWD based on biological swarm rules is a pre-processing method for a non-stationary signal. The most important step of the SWD is swarm filtering (SWF) that operates with the swarm-prey hunting approach and produces oscillatory components (OCs) from an element input data. It has an iterative performing of a sifting-like operating. The prevailing OCs are randomly defined, and then OCs are calculated by using consecutive applications of SWF. The aligned OCs are subtracted from the input data of the rest of the elements Finally, if the residual input data do not include the oscillation mode of adequate energy, the SWD algorithm is finished [15].

The pseudo-code is given in Algorithm 1.

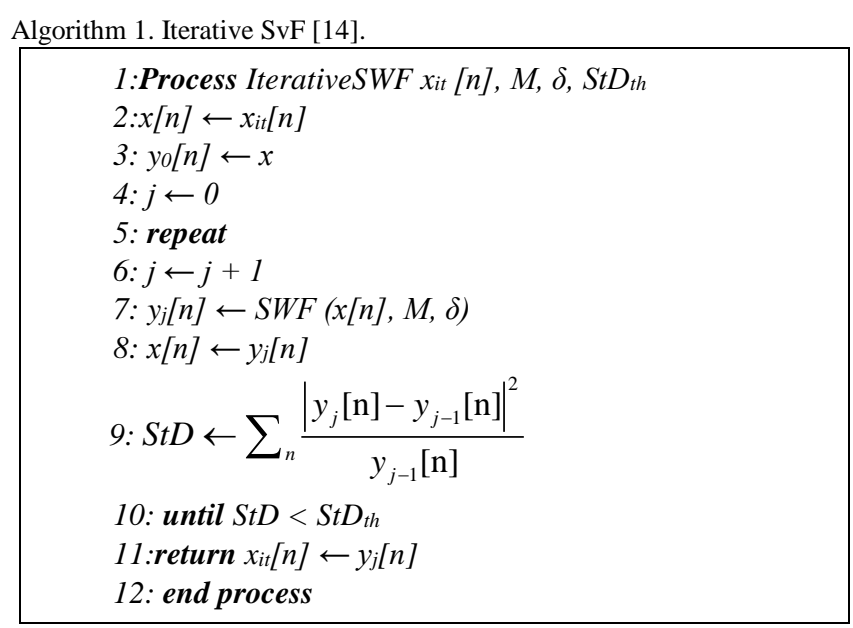

$x[n]$ represents the input signal. $M, \delta$, and $S t D_{t h}$ are the swarm hunting parameters that are members, control flexibility of swarm, and threshold, respectively. $y[n]$ is output of SWF.

Although this pre-processing method has been used for the decomposition of many biomedical signals [16], [17] and in research of different non-stationary signals [18], [19], it has been applied to solar data for the first time in this study.

In this study, SWD is applied to decomposition of the real solar power output data obtained from Akgul-SPP for preprocessing (Fig. 2).

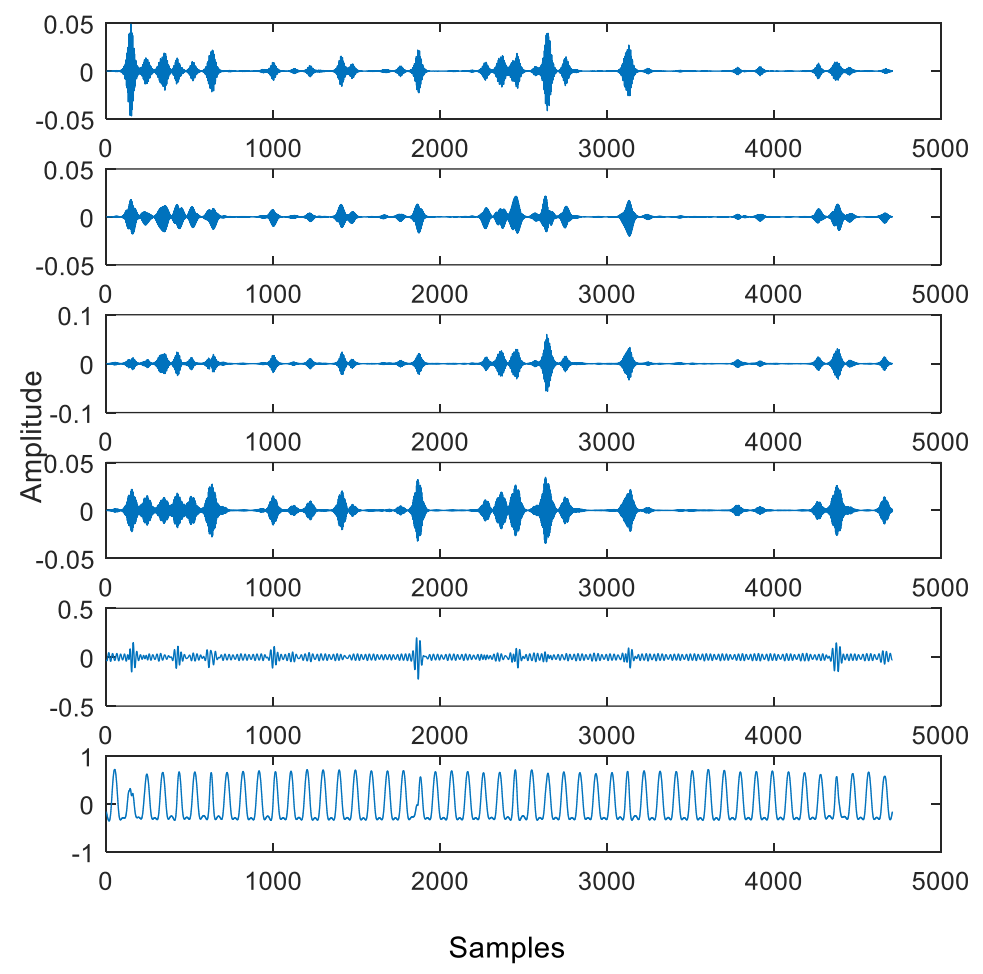

Fig. 2. Results of solar power data decomposition using SWD.

Real solar PV power output time series data of SPP were decomposed into five sub-series by SWD. Particularly high frequency components, which have changes in solar power output during cloudy periods, have been detected in these sub-series. Solar data and reconstructed solar data after the pre-processing are given in Fig. 3. 


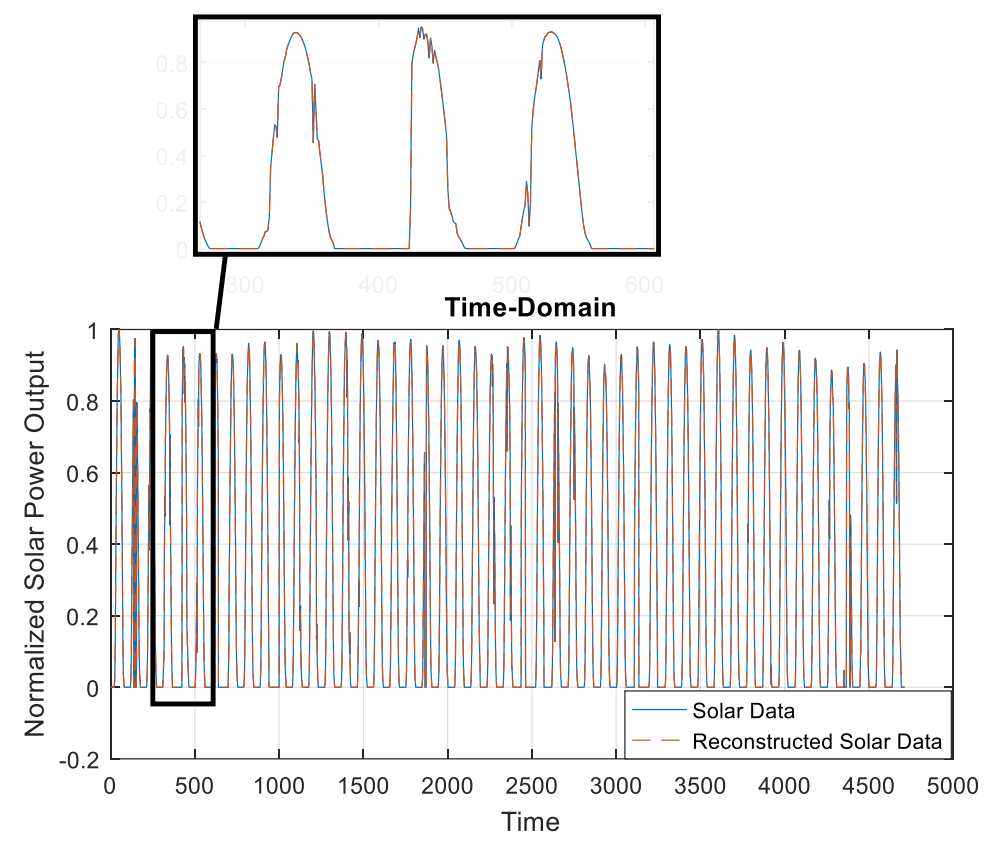

(a)

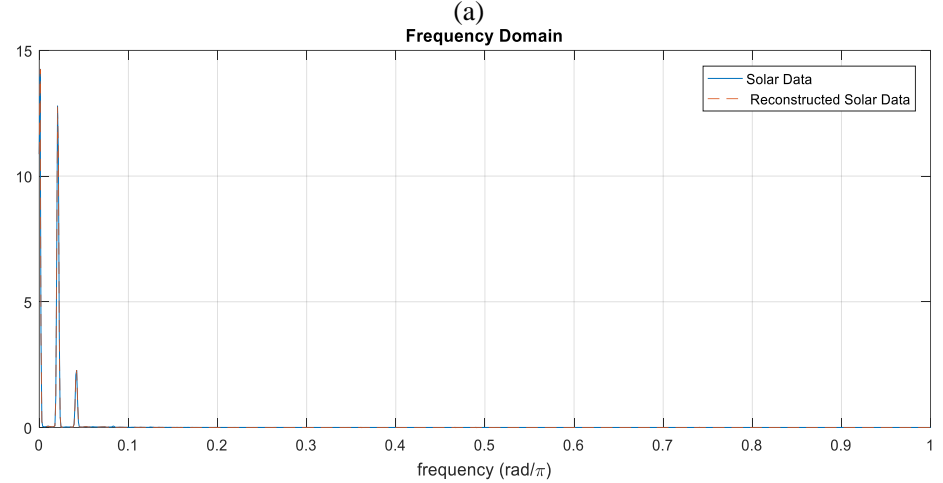

(b)

Fig. 3. The normalized real data and reconstructed signal (a) time and (b) frequency domain (15 min resolution).

\section{SWD ForeCAST MODEL DEVELOPMENT - A CASE STUDY AT AKGUL-SPP}

The total installed capacity of solar PV plant at AkgulSPP is $857.08 \mathrm{kWp}$. It was installed in October 2018 in Elazig, Turkey. The total amount of energy generated since the date of installation is $1,575,116.39 \mathrm{kWh}$. Figure 4 shows the sample hourly power and energy outputs of the power plant for two days.

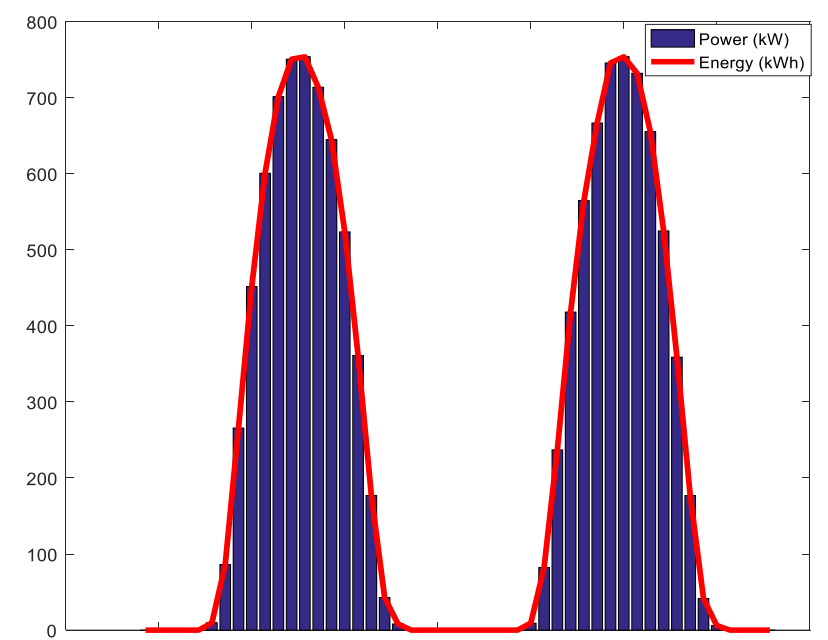

Fig. 4. Power and energy output for 17/07/2019-18/07/2019 time horizon.
The clear sky and cloudy/rainy weather data are chosen from July-August 2019 dataset. The data of July 2019 are used for model development, and that of August 2019 - for testing purpose.

All data were normalized using (1) before pre-processing and steps of analysis

$$
\text { normalize }\{x[n]\}=\frac{[x[n]-\min (x[n])]}{[\max (x[n])-\min (x[n])]} .
$$

The flow chart of the study is given in Fig. 5. Firstly, the data (with 15 min resolution) were collected from SPP and normalized before the pre-processing step. Sub-series of solar data were obtained using SWD technique. For each sub-series, FFNN model was established and outputs were obtained. Separate forecasting results achieved by each subseries models were collected to obtain the value of final estimation. The sliding window technique was used for the forecasting step. Lag matrix data for input were realized in the neural network training and forecasting step.

The hybrid approach (SWD-FFNN) was run 10 times independently. The performance results are evaluated statistically for training and test phases of models. Simulation results and comparative analysis are given in Section IV. 


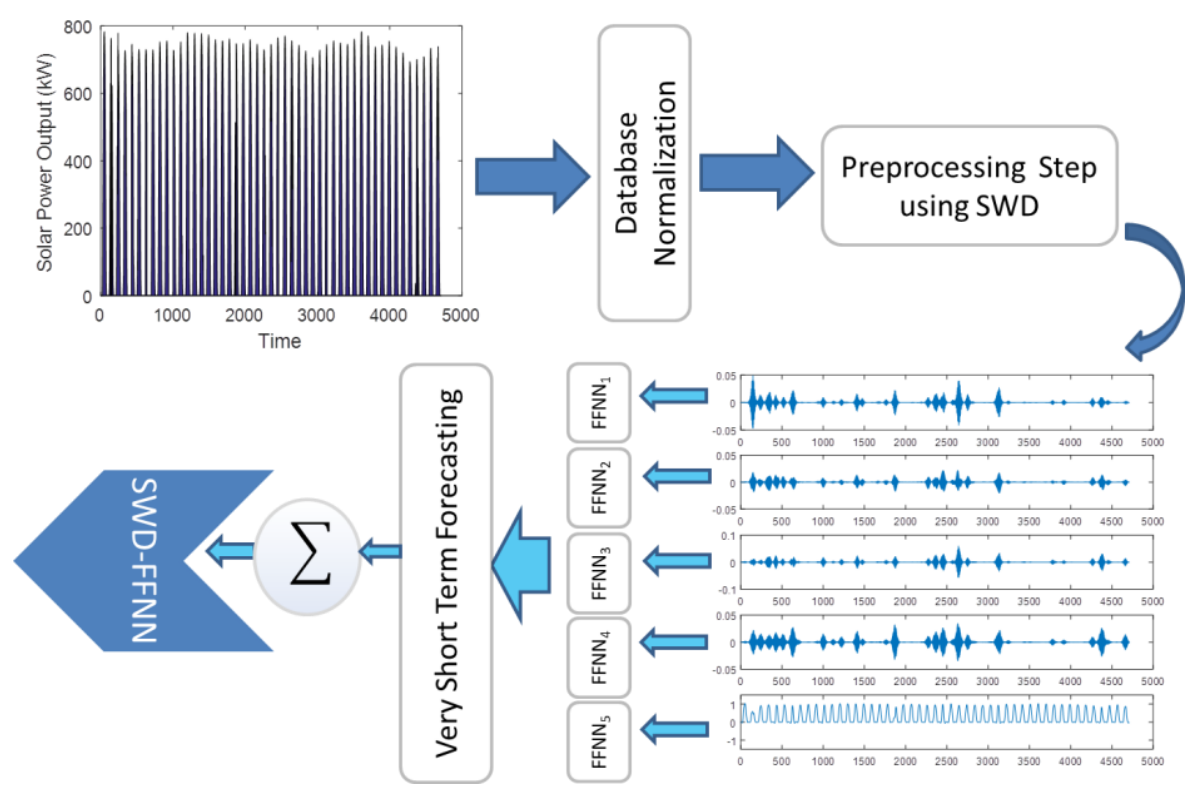

Fig. 5. Flowchart of the SWD-FFNN approach.

$$
\begin{aligned}
& R M S E=\sqrt{\frac{\sum_{i=1}^{N}\left(x_{i}[\mathrm{n}]-\hat{\mathrm{x}}_{i}[\mathrm{n}]\right)^{2}}{N},} \\
& M S E=\frac{1}{N} \sum_{i=1}^{N}\left(x_{i}[\mathrm{n}]-\hat{\mathrm{x}}_{i}[\mathrm{n}]\right)^{2},
\end{aligned}
$$
algorithm. For all sub-series data, different number of neurons and models were analyzed for the first forecasting
step. Finally, accurate forecasting models were obtained for sub-series estimation separately. Figure 6 shows decomposed signal-1 forecasting results and performance metrics statistically.

Some error metrics, such as RMSE (Root Mean Square Error) and MSE (Mean Square Error), were used to evaluate the forecasting performances of the proposed model.

The formulation of the used performance metrics is given as follows:

where $x_{i}[\mathrm{n}]$ and $\hat{\mathrm{x}}_{i}[\mathrm{n}]$ represent observed and estimation values of the solar data, $N$ is the total number of data determined for performance metrics. The reason for using RMSE/MSE is that they express the overall error in solar power output data for the entire data set.

Comparative results of the hybrid model SWD-FFNN and FFNN for all data are given in Fig. 7. Numbers of training and testing data are 3295 and 1412 , respectively.

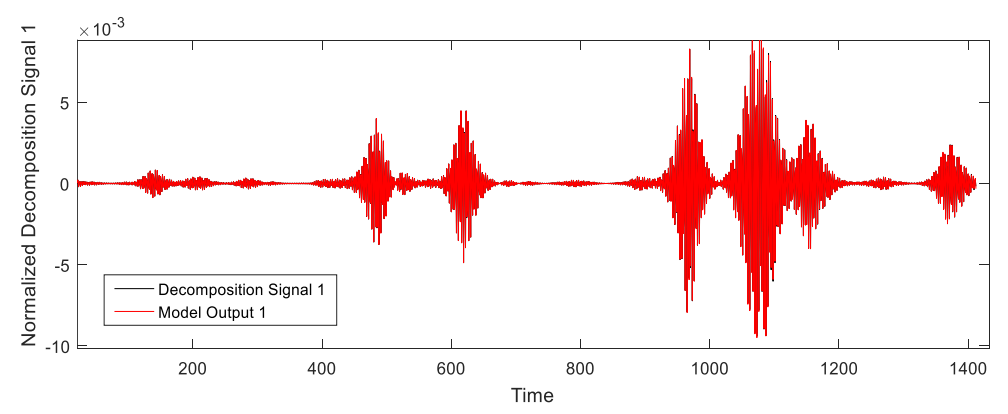

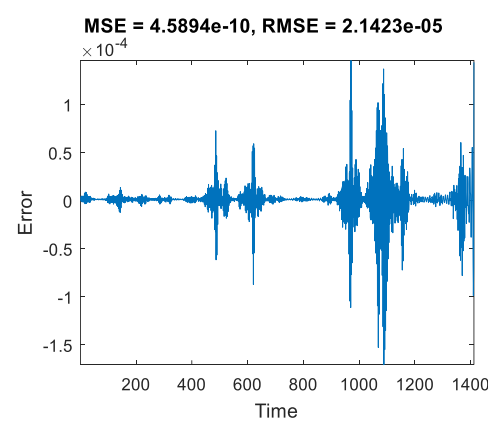

(b)

(a)

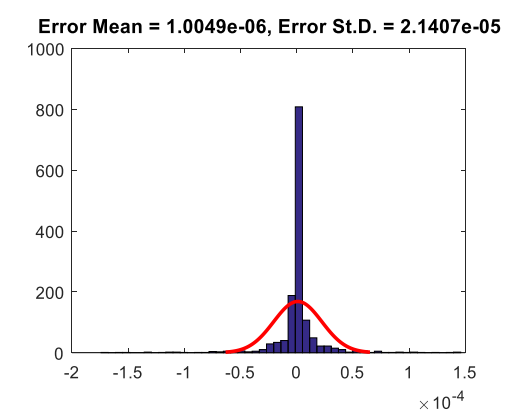

(c)

Fig. 6. The sample results (a) a decomposed signal-1 forecasting (b) error (c) the standard deviation and distribution of error. 


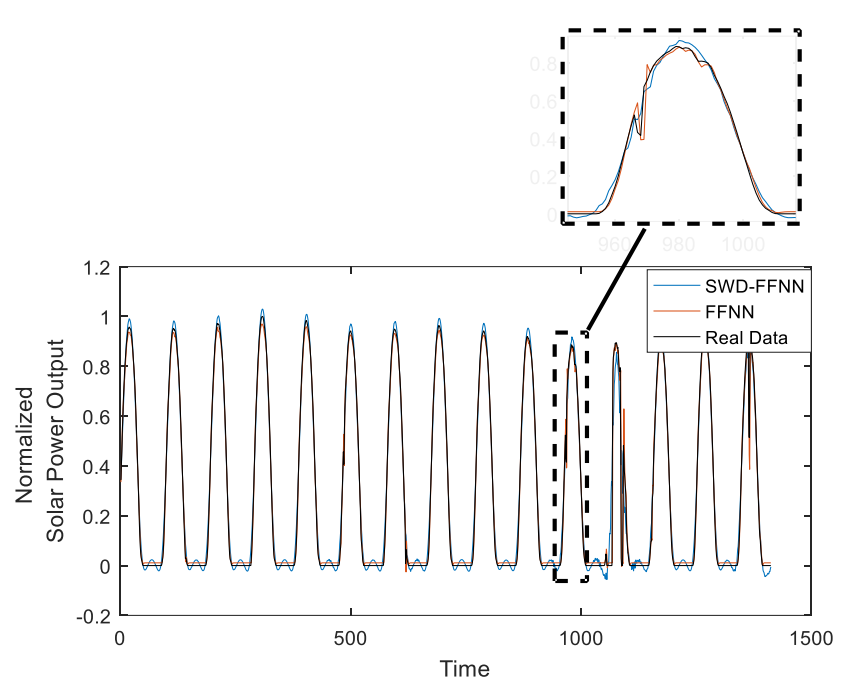

Fig. 7. Hybrid forecasting results.

According to Fig. 7, the performance of the proposed hybrid approach is higher in cloudy periods. According to the results of performance metrics presented in Table I, there is a significant improvement in error measures compared to the other forecast model in selected cloudy case.

TABLE I. COMPARATIVE FORECASTING RESULTS OF PERFORMANCE METRICS IN THE CLOUDY/RAINY SKY CONDITIONS

\begin{tabular}{|c|c|c|}
\hline Performance Criteria & SWD-FFNN & FFNN \\
\hline RMSE & 0.0362 & 0.0512 \\
\hline MSE & 0.0013 & 0.0026 \\
\hline
\end{tabular}

SWD-FFNN approach appears to be more accurate than FFNN in high-frequency components under cloudy and rainy sky conditions although the FFNN model has higher accuracy when forecasting smooth data due to observations.

\section{CONCLUSIONS}

In this paper, a novel PV forecasting model based on SWD-FFNN is given to forecast the power output of a PV system. The chosen data sets are 15 min observations from one particular station in Akgul-SPP, Turkey. Firstly, original PV output data sets were decomposed into five sub-series by SWD. The results were obtained by applying separate estimation models for all sub-series. The simulation results showed that the proposed PV power forecasting model can significantly improve the forecasting performance in cloudy periods compared to other PV forecasting method without pre-processing approach.

\section{CONFLICTS OF INTEREST}

The author declares that he has no conflicts of interest.

\section{REFERENCES}

[1] S. Sobri S. Koohi-Kamali, and N. A. Rahim, "Solar photovoltaic generation forecasting methods: A review", Energy Conversion and Management, vol. 156, pp. 459-497, 2018. DOI 10.1016/j.enconman.2017.11.019.

[2] G. M. YagliD. Yang, and D. Srinivasan, "Automatic hourly solar forecasting using machine learning models", Renewable and Sustainable Energy Reviews, vol. 105, pp. 487-498, 2019. DOI: 10.1016/j.rser.2019.02.006.

[3] C. Wan et al., "Photovoltaic and solar power forecasting for smart grid energy management", CSEE Journal of Power and Energy Systems, vol. 1, no. 4, pp. 38-46, 2015. DOI 10.17775/CSEEJPES.2015.00046.

[4] A. Andrijanovits, H. Hoimoja, and D. Vinnikov, "Comparative review of long-term energy storage technologies for renewable energy systems", Elektronika ir Elektrotechnika, vol. 118, no. 2, pp. 21-26, 2012. DOI: 10.5755/j01.eee.118.2.1168

[5] M. AlKandari and I. Ahmad, "Solar power generation forecasting using ensemble approach based on deep learning and statistical methods", Applied Computing and Informatics, 2019, in press. DOI: 10.1016/j.aci.2019.11.002

[6] Y. Sun, V. Venugopal, and A. R. Brandt, "Short-term solar power forecast with deep learning: Exploring optimal input and output configuration", Solar Energy, vol. 188, pp. 730-741, 2019. DOI: 10.1016/j.solener.2019.06.041

[7] A. T. Eseye, J. Zhang, and D. Zheng, "Short-term photovoltaic solar power forecasting using a hybrid Wavelet-PSO-SVM model based on SCADA and Meteorological information", Renewable Energy, vol. 118, pp. 357-367, 2018. DOI: 10.1016/j.renene.2017.11.011.

[8] V. Kushwaha and N. M. Pindoriya, "A SARIMA-RVFL hybrid model assisted by wavelet decomposition for very short-term solar PV power generation forecast", Renewable Energy, vol. 140, pp. 124-139, 2019. DOI: 10.1016/j.renene.2019.03.020.

[9] M. Rana and A. Rahman, "Multiple steps ahead solar photovoltaic power forecasting based on univariate machine learning models and data re-sampling", Sustainable Energy, Grids and Networks, vol. 21, p. 100286, 2020. DOI: 10.1016/j.segan.2019.100286.

[10] X. Yao, Z. Wang, and H. Zhang, "A novel photovoltaic power forecasting model based on echo state network", Neurocomputing, vol. 325, pp. 182-189, 2019. DOI: 10.1016/j.neucom.2018.10.022.

[11] L. A. Fernandez-Jimenez et al., "Short-term power forecasting system for photovoltaic plants", Renewable Energy, vol. 44, pp. 311-317, 2012. DOI: 10.1016/j.renene.2012.01.108.

[12] J. Shi, W.-J. Lee, Y. Liu et al., "Forecasting power output of photovoltaic systems based on weather classification and support vector machines", IEEE Trans. Ind. Appl., vol. 48, no. 3, pp. 1064 1069, 2012. DOI: 10.1109/TIA.2012.2190816.

[13] JG da Silva Fonseca Jr. et al., "Use of support vector regression and numerically predicted cloudiness to forecast power output of a photovoltaic power plant in Kitakyushu, Japan", Progress in photovoltaics: Research and applications, vol. 20, no. 7, pp. 874882, 2012. DOI: 10.1002/pip.1152.

[14] G. K. Apostolidis and J. L. Hadjileontiadis, "Swarm decomposition A novel signal analysis using swarm intelligence", Signal Processing, vol. 132, pp. 40-50, 2017. DOI: 10.1016/j.sigpro.2016.09.004.

[15] L. J. Hadjileontiadis and Z. M. K. Moussavi, "Current techniques for breath sound analysis", in Breath Sounds. Springer, Cham, 2018, pp. 139-177. DOI: 10.1007/978-3-319-71824-8_9.

[16] S. Alnuaimi et al., "Identification of fetal cardiac timing events by swarm decomposition of Doppler cardiogram signal", in Proc. of Computing in Cardiology Conference (CinC), 2018. DOI: 10.22489/CinC.2018.310

[17] V. Baltatzis et al., "Bullying incidences identification within an immersive environment using HD EEG-based analysis: A swarm decomposition and deep learning approach", Scientific reports, vol. 7, article no. 17292, 2017. DOI: 10.1038/s41598-017-17562-0.

[18] A. Bhattacharyya, L. Singh, and R. B. Pachori, "Fourier-Bessel series expansion based empirical wavelet transform for analysis of nonstationary signals", Digital Signal Processing, vol. 78, pp. 185-196, 2018. DOI: $10.1016 /$ j.dsp.2018.02.020.

[19] Y. Miao et al., "Optimal swarm decomposition with whale optimization algorithm for weak feature extraction from multicomponent modulation signal", Mechanical Systems and Signal Processing, vol. 122, pp. 673-691, 2019. DOI: 10.1016/j.ymssp.2018.12.034. 